\title{
Demodex sp. y dermatosis faciales en pacientes atendidos por consulta externa en un hospital de Chiclayo, Perú, 2015
}

\author{
Katya M. Mera-Villasis 1,ab, Mayra Y. Valderrama-Ayén 1,a, \\ Franklin R. Aguilar-Gamboa ${ }^{1 a}$
}

\section{RESUMEN}

Objetivo. Identificar Demodex sp. y establecer su asociación con las dermatosis faciales (Acné vulgar, Rosácea, Blefaritis y Dermatitis atípica), y otros factores. Material y Métodos. Estudio observacional tipo casos y controles. Se evaluaron 104 pacientes atendidos por consulta externa en el Hospital Regional Lambayeque entre setiembre 2014 a diciembre 2015. De los 104 pacientes atendidos (52 controles y 52 con las patologías estudiadas), se obtuvo muestras de piel del rostro a través de la técnica de biopsia superficial de piel así como la extracción directa de pestañas. Resultados: Se identificó a Demodex sp. ( $\geq 5$ parásitos/cm2) en el $61,5 \%$ de pacientes. Las dermatosis faciales fueron: rosácea $(40,4 \%)$, acné $(36,5 \%)$, dermatitis atípica $(13,5 \%)$ y blefaritis $(9,6 \%)$. Demodex sp. se asoció a dermatitis atípica $(87,5 \%$ de casos , OR=164), rosácea $(71,4 \%, \mathrm{OR}=15)$, blefaritis $(66,7 \%, \mathrm{OR}=16)$, y acné $(50,0 \%$, OR=10). Demodex $s p$. se asoció, en orden decreciente, a mejillas, pestañas, frente, barbilla y nariz. Conclusiones. Demodex $s p$. fue identificado con alta frecuencia y se asoció a dermatosis faciales en la población de estudio. Se recomienda la vigilancia e investigación de esta acarosis en el diagnóstico diferencial en población de riesgo..

Palabras clave: Acaro, Dermatosis Facial, Acné Vulgar, Rosácea, Blefaritis, Dermatitis (Fuente: DeCS- BIREME).

\footnotetext{
1. Laboratorio de Inmuno-virología del Hospital Regional Lambayeque, Lambayeque, Perú.

a. Biólogo Microbiólogo.

b. Maestro en Ciencias.
} 


\section{Demodex sp. and facial dermatosis in patients seen by outpatient cli- nic in a hospital in Peru}

\section{RESUMEN}

Objective. Identify Demodex sp. and establish its association with facial dermatosis (acne vulgaris, rosacea, blepharitis and atypical dermatitis), and other factors. Material and Methods. Observational study of cases and controls. 104 patients attended by external consultation at the Hospital Regional Lambayeque between September 2014 and December 2015 were evaluated. Of the 104 patients treated (52 controls and 52 with the pathologies studied), skin samples of the face were obtained through the superficial skin biopsy technique as well as the direct extraction of eyelashes. Results. Demodex $s p$. $(\geq 5$ parasites $/ \mathrm{cm} 2)$ in $61.5 \%$ of patients, the identified species was $D$. folliculorum. Facial dermatoses were: rosacea $(40.4 \%)$, Acne $(36.5 \%)$, atypical dermatitis (13.5\%) and blepharitis (9.6\%). Demodex $s p$. it was associated with atypical dermatitis $(87.5 \%$ of cases, OR $=164)$, rosacea $(71.4 \%$, OR $=15)$, blepharitis $(66.7 \%, \mathrm{OR}=16)$, and acne $(50.0 \%$, OR $=10)$. Demodex $s p$. it was associated, in descending order, with cheeks, eyelashes, forehead, chin and nose. Conclusions. Demodex $s p$. It was identified with high frequency and associated with cases of facial dermatoses in the study population. Surveillance and investigation of this acarosis is recommended in the differential diagnosis in the population at risk.

Key words: Demodex sp.., Acne, Rosacea, Blepharitis, atipic dermatitis (source: MeSH-NLM).

\section{INTRODUCCIÓN}

En la piel humana es posible encontrar una variedad de microorganismos, la mayoría de ellos se encuentran en estado de colonización, y solo en condiciones particulares pueden causar daño (1); en este grupo de organismos encontramosdesde bacterias hasta artrópodos como es el caso de Demodex spp. Existen más de 100 especies de Demodex, en su mayoría son comensales obligados de las unidades pilosebáceas de los mamíferos; pero dos especies se reconocen como ectoparásitos frecuentes en humanos. Estos son considerados parásitos oportunistas o vectores de hongos y bacterias (2-4): D. folliculorum, que se encuentra en los folículos pilosos, y $D$. brevis que se localiza dentro de las glándulas sebáceas ${ }^{(5,6)}$.

El potencial patogénico de Demodex sp. es controvertido, para algunos investigadores la presencia de estos parásitos en la piel humana es muy frecuente, llegando a ser $100 \%$ en adultos de edad avanzada (2,7). En efecto, se plantea que la densidad parasitaria sería el factor determinante entre el desarrollo de problemas dermatológicos y el estado portador sano. Sin embargo, para otros investigadores, Demodex constituye un patógeno per se y como vector de bacterias causantes de enfermedades cuya vía de infestación se desconoce; aunque se postula su transmisión por contacto directo entre las personas ${ }^{(8)}$.

Estudios previos demuestran que la multiplicación de los ácaros en las superficies corporales ocasionan varios tipos de dermatosis faciales; siendo reconocido por muchos investigadores como una importante causa de enfermedades de la piel y como un problema de salud pública ${ }^{(5,7,7,9)}$. Así mismo, se ha descrito la asociación de la acarosis con diferentes patologías ${ }^{(4,12)}$, rosácea ${ }^{(10,13)}$, SIDA ${ }^{(14)}$, blefaritis ${ }^{(5,15,16)}$, foliculitis ${ }^{(12)}$ y acné vulgar ${ }^{(11,17)}$; generando en los pacientes, principalmente en los jóvenes, un estrés mental y trastornos psicológicos ${ }^{(10,11,18)}$.

En el Perú, y más aún en la región Lambayeque, las investigaciones sobre este parásito son reducidas y su conocimiento limitado. Por tanto es necesario investigar la presencia y densidad parasitaria de Demodex sp. con la finalidad de fortalecer el diagnóstico de las dermatosis faciales; las cuales actualmente están unidas a la salud mental, contribuyendo en la disminución de la autoestima personal e influyendo negativamente en la participación activa de la persona en la vida social. El objetivo de este estudio fue identificar Demodex sp. y describir su asociación con las dermatosis faciales (acné vulgar, rosácea, blefaritis y dermatitis atípica), la edad, sexo y tenencia de mascotas, eosinofilia, incremento de IgE e infección por el virus de la inmunodeficiencia humana $(\mathrm{VIH})$..

\section{MATERIAL Y MÉTODOS}

\section{Tipo de investigación}

Estudio observacional analítico tipo casos y controles.

Población y muestra

La población fueron los pacientes atendidos por con- 
sulta externa de los servicios de infectología y oftalmología del Hospital Regional Lambayeque (HRL) en Chiclayo, Perú, entre setiembre 2014 y diciembre del 2015. Hospital de alta complejidad, categoría III-1, que atiende pacientes referidos de la región Lambayeque y regiones aledañas ${ }^{(19)}$. Se incluyeron a pacientes mayores de 12 años de edad con rosácea, acné vulgar, blefaritis y dermatitis atípicas; y se excluyeron a los pacientes que habían recibido algún tipo de medicamento acaricida al momento o días previos a la toma de muestra.

La muestra fue no probabilística y comprendió a 104 pacientes, de los cuales 52 presentaron algún tipo de dermatosis (blefaritis, acné, rosácea y dermatitis atípica), y 52 pacientes que no presentaron ninguna de las dermatosis estudiadas (controles). Así mismo, el método de muestreo fue aleatorio simple.

\section{Técnicas e instrumentos de recolección datos}

\section{Biopsia cutánea superficial estandarizada}

Este es un método diagnóstico no invasivo que permite obtener la superficie del estrato corneo y el contenido del folículo piloso (1). La técnica consistió en marcar en una lámina portaobjeto, un cuadrado de $1 \mathrm{~cm} 2$ de lado (con plumón indeleble); se aplicó en el centro del cuadrado una gota del adhesivo de cianocrilato "Super glue". Se colocó la lámina con el pegamento sobre la superficie cutánea a evaluar (mejilla, frente, barbilla, nariz). Después de aproximadamente dos minutos se despegó la lámina portaobjetos conteniendo el pegamento, en el cual quedó adherido el estrato corneo y los folículos pilosos. Se aplicó a la muestra obtenida una gota de aceite de inmersión (para aclaramiento) y luego se colocó la lámina cubreobjetos.

La cantidad de parásitos se determinó contando el número de Demodex sp. dentro del cuadrado de $1 \mathrm{~cm} 2$ previamente marcado ${ }^{(2)}$. Se consideró significación clínica un recuento mayor o igual a 5 parásitos por centímetro cuadrado ${ }^{(2,20)}$.

\section{Extracción de pestañas en casos de blefaritis}

Se realizó usando una pinza para depilar cejas. Se extrajeron tres pestañas de cada párpado (sup. der., sup. izq., inf. der., inf. izq.), incidiendo en las zonas como las comisuras palpebrales, especialmente en el párpado inferior. Las pestañas extraídas se colocaron en láminas portaobjeto y sobre ellas se agregó una gota de aceite de inmersión para aclarar al ácaro; luego se cubrió con una lámina cubreobjetos e inmediatamente se procedió a observar en el microscopio $(3,5,15)$. Se consideró infestación desde un ectoparásito por pestaña, para el caso de blefaritis.

\section{Identificación taxonómica de Demodex sp.}

Se realizó según la clave taxonómica descrita por Godinez et al $2004^{(4)}$, donde se consideraron características morfológicas del estadío adulto como del huevo.
Medición de las características clínicas y epidemiológicas

La eosinofilia se realizó mediante el recuento de eosinófilos en frotis de sangre según descrito en el "Manual de procedimientos de laboratorio en técnicas básicas de hematología (Norma $N^{\circ}$ 40-INS)". Asimismo, el dosaje de IgE se realizó mediante la técnica de Elisa cuantitativa, siguiendo las recomendaciones del fabricante (MONOBIND, USA). El kit indica una sensibilidad analítica de 1.0 $\mathrm{UI} / \mathrm{ml}$ y no ninguna una reacción cruzada con los demás tipos de anticuerpos. Los valores superiores a $200 \mathrm{UI} / \mathrm{ml}$ se interpretaron como niveles altos. Las demás características epidemiológicas y clínicas (edad, sexo, procedencia, grado de instrucción, ocupación, tipo de dermatosis facial, tenencia de mascotas en casa), fue realizada a través de un cuestionario estructurado, aplicado por personal capacitado.

\section{Análisis de datos}

Los datos fueron organizados por cada factor evaluado en tablas de contingencia donde se presentaron las frecuencias de las categorías de cada variable independiente (factor), una prueba inferencial correspondiente (Chi cuadrado) para evaluar asociación poblacional entre las variables consideradas y Demodex sp..; a su vez, fueron calculados los Odds ratio (OR). Se consideró un nivel de confianza del $95 \%$ y un valor de $p<0,05$ como significativo. Se utilizó el SPSS versión 23 y el Epidat 3.1.

\section{Consideraciones éticas}

Para la realización del trabajo se contó con la aprobación del Comité de Investigación y Ética del HRL. La participación en el estudio fue voluntaria y aceptada a través de la firma de un consentimiento informado por parte del padre o apoderado y un asentimiento informado por los niños mayores de 12 años.

\section{Resultados}

En el estudio realizado desde setiembre del 2014 a diciembre del 2015, se evaluaron pacientes desde los 12 años a más de 60 años de edad encontrándose que del total de pacientes atendidos en consultorio externo del HRL, el $61,5 \%$ de pacientes con dermatosis estuvieron infestado por Demodex sp. en una densidad $\geq 5$ parásitos $/ \mathrm{cm} 2$, mientras que sólo el $11,5 \%$ del grupo control estaban infestados con dicho parásito.

El análisis estadístico reveló una variación significativa ( $p$ $<0,001$ ) entre ambos grupos, es decir, los pacientes con Demodex $s p$. a una densidad $\geq 5 / \mathrm{cm} 2$, presentaron 12 veces más probabilidad de presentar una dermatosis facial, en comparación a los controles. Asimismo, la frecuencia y la asociación entre las características clínicas y epidemiológicas con la presencia de Demodex $s p$. (densidad $\geq 5$ / 
cm2), se presentan en la tabla 1. En cuanto a la distribución de Demodex sp. en el rostro, se observó mayor probabilidad de presentación del parásito en las mejillas $(p<0,001)$, pestañas $(p<0,001)$ y frente $(p=0,044)$. Ver tabla 2.

Por otro lado, el análisis bivariado entre los tipos de dermato-

Tabla 1. Características de los pacientes admitidos en consulta externa de un hospital del norte del Perú y su asociación con la

infección por Demodex sp., setiembre del 2014 a diciembre del 2015.

\begin{tabular}{|c|c|c|c|c|}
\hline Variable & $\mathbf{N}(\%)$ & $\begin{array}{c}\text { Demodex sp. } \geq 5 \text { parásit- } \\
\text { os } x \mathrm{~cm} 2 \text { /total }\end{array}$ & OR (IC 95\%) & Valor $p$ \\
\hline \multicolumn{5}{|l|}{ Sexo } \\
\hline Femenino & $52(50,0)$ & $17 / 52(32,7)$ & \multirow{2}{*}{$1,39(0,62-3,12)$} & \multirow{2}{*}{$0,541^{*}$} \\
\hline Masculino & $52(50,0)$ & $21 / 52(40,4)$ & & \\
\hline \multicolumn{5}{|l|}{ Grupo etario } \\
\hline $12-28$ años & $48(46,2)$ & $14 / 48(29,2)$ & 1 & \multirow{4}{*}{$0,294^{* *}$} \\
\hline 28-46 años & $38(36,5)$ & $15 / 38(39,5)$ & $1,58(0,64-3,91)$ & \\
\hline 46-64 años & $12(11,5)$ & $5 / 12(41,7)$ & sd & \\
\hline > 64 años & $6(5,8)$ & $4 / 6(66,7)$ & sd & \\
\hline \multicolumn{5}{|l|}{ Dermatosis } \\
\hline $\mathrm{Si}$ & $52(50,0)$ & $32 / 52(61,5)$ & \multirow{2}{*}{$12,27(4,43-33,94)$} & \multirow{2}{*}{$<0,001$ * } \\
\hline No & $52(50,0)$ & $6 / 52(11,5)$ & & \\
\hline \multicolumn{5}{|c|}{ Pacientes con animales en casa } \\
\hline Sí & $47(45,2)$ & $20 / 47(42,6)$ & \multirow{2}{*}{$1,61(0,71-3,59)$} & \multirow{2}{*}{$0,341^{*}$} \\
\hline No & $57(54,8)$ & $18 / 57(31,6)$ & & \\
\hline \multicolumn{5}{|l|}{$\operatorname{lgE}>200 \mathrm{Ul}$} \\
\hline Sí & $38(38)$ & $12 / 38(31,6)$ & \multirow[t]{2}{*}{$0,73(0,31-1,72)$} & \multirow[t]{2}{*}{$0,612^{*}$} \\
\hline No & $62(62)$ & $24 / 62(38,7)$ & & \\
\hline \multicolumn{5}{|l|}{ Eosinofilia } \\
\hline Sí & $31(31.96)$ & $17 / 31(54,8)$ & \multirow[t]{2}{*}{$3,00(1,25-7,21)$} & \multirow[t]{2}{*}{$0,024^{*}$} \\
\hline No & $66(68,04)$ & $19 / 66(28,8)$ & & \\
\hline \multicolumn{5}{|l|}{ Infección VIH } \\
\hline Sí & $29(27,9)$ & $10 / 29(34,5)$ & \multirow[t]{2}{*}{$0,88(0,36-2,18)$} & \multirow[t]{2}{*}{$0,965^{\star}$} \\
\hline No & $75(72,1)$ & $28 / 75(37,3)$ & & \\
\hline
\end{tabular}

(*) Prueba exacta de Fisher. $\left.{ }^{* *}\right)$ Prueba de Chi cuadrado.. sis faciales y la presencia de Demodex sp., demostró que todos los tipos alta probabilidad de presentarse cuando Demodex sp. estuvo presente $(p<0,001)$, ver tabla 3 . 
Tabla 2. Ubicación de las dermatosis faciales y su asociación con la infección por Demodex sp. en pacientes admitidos en consulta externa de un hospital del norte del Perú, setiembre del 2014 a diciembre del $2015 .$.

\begin{tabular}{|c|c|c|c|}
\hline Variable & $\begin{array}{l}\text { Demodex sp. } \geq 5 \\
\text { parásitos } x \mathrm{~cm} 2 / \text { total }\end{array}$ & OR (IC 95\%) & Valor $p^{x}$ \\
\hline \multicolumn{4}{|l|}{ Mejilla } \\
\hline Dermatosis & $29 / 52(55,8)$ & $31,52(6,92-143,4)$ & $<0,001$ \\
\hline Controles & $2 / 52(3,8)$ & & \\
\hline \multicolumn{4}{|l|}{ Frente } \\
\hline Dermatosis & $11 / 52(21,2)$ & $4,38(1,14-16,77)$ & 0,044 \\
\hline Controles & $3 / 52(5,8)$ & & \\
\hline \multicolumn{4}{|l|}{ Barbilla } \\
\hline Dermatosis & $7 / 52(13,5)$ & $3,33(0,75-14,72)$ & 0,184 \\
\hline Controles & $2 / 52(3,8)$ & & \\
\hline \multicolumn{4}{|l|}{ Nariz } \\
\hline Dermatosis & $3 / 52(5,8)$ & $2,43(0,35-17,09)$ & 0,618 \\
\hline Controles & $1 / 52(1,9)$ & & \\
\hline \multicolumn{4}{|l|}{ Pestaña } \\
\hline Dermatosis & $21 / 52(40,4)$ & $5,19(1,88-14,33)$ & $<0.001$ \\
\hline Controles & $6 / 52(11,5)$ & & \\
\hline
\end{tabular}

(*) Calculado mediante prueba exacta de Fisher.

Tabla 3. Tipos de dermatosis faciales y su asociación con la infección por Demodex sp. en pacientes admitidos en consulta externa de un hospital del norte del Perú, setiembre del 2014 a diciembre del 2015.

\begin{tabular}{lcccc}
\hline \multicolumn{1}{c}{ Variable } & N (\%) & $\begin{array}{c}\text { Demodex sp. } \geq 5 \\
\text { parásitos } \mathrm{x} \mathrm{cm2} \mathrm{I} \\
\text { total }\end{array}$ & OR (IC 95\%) & Valor $\mathbf{p}^{*}$ \\
\hline Rosácea & $21(33,3)$ & $\begin{array}{c}15 / 21(71,4) \\
\mathrm{Si}\end{array}$ & $15(4,16-54,05)$ & $<0,001$ \\
$\mathrm{No}$ & $42(66,7)$ & $6 / 42(14,3)$ & & \\
Acné vulgar & $22(33,3)$ & $11 / 22(50,0)$ & $10(2,66-37,61)$ & $<0,001$ \\
$\mathrm{Si}$ & $44(66,7)$ & $4 / 44(9,1)$ & & \\
No & & & & \\
& & & & \\
Blefaritis & $12(25,0)$ & $8 / 12(66,7)$ & $16(3,27-78,28)$ & $<0,001$ \\
$\mathrm{Si}$ & $36(75,0)$ & $4 / 36(11,1)$ & & $<0,001$ \\
No & & & $161(8,88-$ & \\
Dermatitis atípica & $8(25)$ & $7 / 8(87,5)$ & $2920,3)$ & \\
Si & $24(75)$ & $1 / 24(4,2)$ & & \\
No & & & & \\
\hline \hline
\end{tabular}




\section{DISCUSION}

Demodex $s p$. es un parásito vinculado a los problemas dérmicos, principalmente en cara y cuello. En esta investigación, el frecuencia de positividad a la presencia del parásito en pacientes con dermatosis $(65,5 \%)$ es alto, de tal forma que el parásito está positivamente asociado a la enfermedad; dicho porcentaje es mayor que el detectado en una investigación por J. Bikowski et al. (2009), cuyos pacientes $(50 \%)$ mostraron enrojecimiento facial y erupciones sin resolver ${ }^{(8)}$. Pero es muy similar al reportado por otro estudio por Dolenc-Voljc et al. (2005) (62,2\%) (21); esto se explica en el hecho de que ambos estudios desarrollaron la misma metodología (biopsia cutánea superficial estandarizada, BCS) y se encontró la misma densidad parasitaria ( $\geq 5$ parásitos $/ \mathrm{cm} 2$ ).

La presencia de Demodex sp.. como factor de riesgo de dermatosis en pacientes atendidos en consultorio externo del HRL se presentó en el $65,5 \%$ de los casos con un OR de 12,27 mayor al reportado por dos investigaciones $(4,22)$, debido probablemente a que éste estudio involucró casos de rosácea, blefaritis, acné y dermatitis atípica (caracterizada por picazón facial intensa sin causa aparente), mientras que los autores sólo a casos de dermatits y, como se ha tratado anteriormente, los casos en relación de Demodex sp.. con rosácea es bastante alto lo que eleva la prevalencia global.

En relación a la especie de Demodex sp., la única especie encontrada en los caso de dermatosis fue $D$. folliculorum coincidiéndose con varios autores, principalmente porque son casos asociados a blefaritis y rosácea ${ }^{(9,15,23,24)}$.

El mayor número de pacientes evaluados son fueron con diagnóstico de rosácea, habiéndose encontrado en este estudio un $71,4 \%$ de positividad para Demodex sp., OR de 15 y un IC95\% de 4,16-54,05, estas cifras son mayores a las reportados por diferentes investigaciones realizadas ${ }^{(9,10,24)}$. Las diferencias se debe a que los pacientes involucrados en este trabajo tuvieron un amplio rango de edad: entre los 12 y 82 años; quienes presentan mayor variabilidad en las características de la piel, en comparación a sólo adolescente que tienen factores como el cambio hormonal que involucra mayor producción de seborrea fuente de energía del parásito necesaria para su reproducción ${ }^{\left({ }^{17}\right)}$. Así mismo es muy significativo que el OR y la densidad parasitaria sean mucho mayores a las reportadas por los diversos autores; lo que permite reiterar que Demodex sp. tiene un papel patogénico importante en el desarrollo de rosácea ${ }^{(24,25)}$.

El acné representó la segunda causa de dermatitis determinada en ésta investigación, se relacionó con Demodex sp.. en el $50 \%$ de pacientes con acné, con un valor de OR de $10 \mathrm{y}$ un IC95\% de 2,65 a 37,61, estos resultados concuerdan con los obtenidos por dos investigaciones realizadas en China
(11,26), una en relación a los pacientes con infección severa $(\mathrm{OR}=10,3)$, que mostraban un cuadro avanzado de acné que a su vez les ocasionaba una fuerte depresión y la otra investigación realizada con adolescentes de China, en donde el porcentaje de relación Demodex sp. y acné es similar, pero el OR fue significativamente mayor para el presente trabajo.

La asociación Demodex sp. con la dermatitis en esta investigación fue del $87,5 \%$ (infestación por este parásito) con un OR de 161, por tanto, el parásito está altamente asociado a la enfermedad; sin embargo, este resultado no es concordante con lo reportado por una investigación en donde se identificó la presencia de $D$. mitis, especie poco relacionada a dicha parasitosis ${ }^{(22)}$. No obstabte, el mismo resultado coincide con otras investigaciones donde en todos los casos se identificó a $D$. folliculorum ${ }^{(21,27)}$.

En cuanto a la blefaritis, la positividad también fue elevada y los resultados concuerdan con diversos estudios previos ${ }^{(15,16,23)}$. En el presente estudio, Demodex sp. se presentó con mayor frecuencia en las mejillas, seguido de pestañas, frente, barbilla y nariz; al respecto, es probable también la posibilidad de que el uso frecuente de antibacterianos, antialérgicos y esteroides tópicos oculares por los pacientes limite la flora bacteriana y barreras protectoras normales predisponiendo a la infestación parasitaria ${ }^{(20)}$.

En relación al sexo (uniendo dermatosis y controles); los pacientes varones evaluados presentaron un porcentaje mayor de infestación facial por Demodex sp., en comparación con las mujeres. sin embargo al realizar el análisis estadístico mujeres y varones tienen la misma posibilidad de ser infestados por este parásito, así como lo manifiestan algunos trabajos previos con los cuales se coincide $(11,13,16,22,28)$. La presencia de animales en casa también fue evaluada, y, si bien, el análisis estadístico no mostró significación; sí se observó un mayor porcentaje de infección por Demodex sp. en aquellas que poseían una mascota (05,10).

En este estudio, a pesar que las personas infectadas por Demodex sp. no presentaron cantidades de IgE elevada, desarrollaron mayor frecuencia de Eosinofilia en comparación con las personas sin infección por este parásito. $\mathrm{Al}$ respecto, estudios realizados en pacientes con demodicosis ocular mostraron una notable disminución de los síntomas después de la erradicación total de Demodex sp., sugiriendo que este parásito juega un papel patológico o agravante en los trastornos inflamatorios, con respuestas inmunes hipersensibles sobre la superficie ocular (29). 
En cuanto a los pacientes con VIH evaluados, el 34,5\% presentaron Demodex sp., y aunque al realizar el análisis estadístico, no existió una asociación significativa, la literatura manifiesta que este parásito está implicado como causante de foliculitis pruriginosa lo que causaría un problema de salud que podría agravarse en estos pacientes por su estado inmunológico ${ }^{(14)}$.

El estudio presentó limitaciones en el diseño, pues no permitió ajustar los análisis según variables confusoras: sexo, consumo de alcohol, fumar y tipo de piel; sin embargo, los hallazgos mostrados representan una primera aproximación de la comprensión del problema estudiado. Además, el reducido número de participantes, respecto a los programados, también representó una limitación; principalmente debido dificulta para enrolar participantes debido a la reducida cantidad de médicos que investigan esta condición clínica.

Se concluye que, Demodex sp. ( $\geq 5$ parásitos/cm2) presenta alta frecuencia en pacientes con dermatosis facial atendidos en un hospital de Chiclayo. Asimismo, el parásito se asoció a dermatitis atípica, rosácea, a blefaritis y acné, constituyéndose en un factor asociado a dermatosis facial. La mayor localización del parásito, en orden decreciente fue: mejillas, pestañas, frente, barbilla y nariz.

Conflicto de interés: los autores declaran no tener conflictos de interés.

Financiamiento: autofinanciado.

\section{REFERENCIAS BIBLIOGRÁFICAS}

1. Porta Guardia CA, Feijó F. Importancia etiológica de la densidad cutánea del Demodex folliculorum en la Rosácea [Internet]. Tesis doctoral: Universidad Nacional de Rosario. Universidad Naciona de Rosario; 2003. 55p. Disponible en: http://www.dermatologiarosario.com.ar/pps/tesis/TESIS DE DOCTORADOPORTAGUARDIA.pdf

2. Serrano Grau P. Dermatosis inducidas por Demodex folliculorum Piel. 2011; 26(6):291-5.

3. Castellanos HL. Rosacea, Demodex folliculorum y metronidazol tópico. Rev la Fac Med UNC. 1998; 46(4):194-8.

4. Godinez Hana AL, Medina de la Garza CE, Velazquez Arenas L, Garcia Guerrero CJ, Gonzalez Gonzalez SE. Prevalence of Demodex folliculorum and Demodex brevis in a mexican population. Med Univ. 2004; 6(23):96-100.

5. Rodríguez H, Guillen Z, Romero G. Prevalencia de Demodex sp. en pacientes con Blefaritis. An la Fac Med. 2000; 64(4): 299-304.

6. Liu J, Sheha H, Tseng SCG. Pathogenic role of Demodex mites in blepharitis. Curr Opin Allergy Clin Immunol. 2010; 10(5):505-10.

7. Millás Mascarós E. Parasitación por Demodex folliculorum [Internet]. Control Calidad SEIMC. 2005. Disponible en: https://www. seimc.org/contenidos/ccs/revisionestematicas/parasitologia/Demodex.pdf

8. Bikowski JB, Del Rosso JQ. Demodex dermatitis: a retrospective analysis of clinical diagnosis and successful treatment with topical crotamiton. J Clin Aesthet Dermatol. 2009; 2(1):20-5.

9. Zhao YE, Wu LP, Peng Y, Cheng H. Retrospective analysis of the association between Demodex infestation and rosacea. Arch Dermatol. 2010; 146(8):896-902.

10. Trejo Mucha E, Puell Ramirez L, Salomón M, Vidal Anzardo M, Del Solar Chacaltana M, Bravo Puccio F, et al. Demodicidosis en pacientes con rosácea. Rev Medica Hered. 2007; 18(1):15-21.
11. Zhao Y, Guo N, Xun M, Xu J, Wang M, Wang D. Sociodemographic characteristics and risk factor analysis of Demodex infestation (Acari: Demodicidae). J Zhejiang Univ Sci B. 2011; 12(12):998-1007.

12. Corredor-Osorio R, Nava Á, Tovilla JL, Tovilla JL, Muñoz S. Blefaritis por Demodex folliculorum. Rev Fac Med UNAM. 2000; 43(4):125-9.

13. Li J, O'Reilly N, Sheha H, Katz R, Raju VK, Kavanagh K, et al. Correlation between ocular Demodex infestation and serum immunoreactivity to Bacillus proteins in patients with facial rosacea. Ophthalmology. 2010; 117(5):870-877.e1.

14. López-Vélez R. Infección por VIH y enfermedades parasitarias. Enf Emerg. 2003; 5(1):9-12.

15. Sedeño Cruz I, Novoa Sánchez E, Padrón Álvarez V, García Gonzáles F, San Martín Geisse R. Blefaritis por Demodex folliculorum. Diagnóstico y tratamiento. Rev Cuba Oftalmol. 2006; 19(1):1-5.

16. Rodríguez A, Ferrer C, Alió J. Demodex y blefaritis crónica. Arch Soc Esp Oftalmol. 2005; 80(11):635-42.

17. Santamaría V. Acné vulgar o juvenil. Rev Cent Dermatol Pascua. 2000; 9(1):49-56

18. Thiboutot DM. Acne rosacea. Am Fam Physician. 1994; 50(8):16917, 1701-2.

19. Silva-Díaz H, Campos-Flores H, Llagas-Linares JP, LLatas-Cancino D. Coccidiosis intestinal en niños admitidos en un hospital de Perú y comparación de dos métodos para la detección del Cryptosporidium spp. Rev Peru Med Exp Salud Publica. 2016; 33(4):739-44.

20. Chin-Wong JL, Niño-Pecina A, Ruiz-Quintero N, Naranjo-Tackman R. Prevalencia de demodicosis en pacientes con blefaroconjuntivitis crónica multitratada. Rev Mex Oftalmol. 2006; 80(2):61-3.

21. Dolenc-Voljc M, Pohar M, Lunder T. Density of Demodex folliculorum in perioral dermatitis. Acta Derm Venereol. 2005; 85(3):211-5.

22. Tehrani S, Tizmaghz A, Shabestanipour G. The Demodex mites and their relation with seborrheic and atopic Dermatitis. Asian Pac J Trop Med. 2014; 7:S82-4.

23. Galvis-Ramírez V, Tello-Hernández A, Álvarez-Osorio L, Rey-Serrano JJ. Prevalencia de infección por Demodex folliculorum en pacientes que acuden a consulta general de oftalmología. Rev Salud Pública. 2011; 13(6):990-7.

24. Zhao $Y$, Peng $Y$, Wang $X$, Wu L, Wang $M$, Yan $H$, et al. Facial dermatosis associated with Demodex: a case-control study. J Zhejiang Univ Sci B. 2011; 12(12):1008-15.

25. Bauzá Diez ME, Montero Vare M del P, Castilla Huapaya ID, Dager Haber A, Batista Muñoz N. Rosácea. Algunas características clínicas-epidemiológicas. Correo Científico Médico de Holguín. 2006; 10(4):1-4.

26. Zhao Y-E, Hu L, Wu L-P, Ma J-X. A meta-analysis of association between acne vulgaris and Demodex infestation. J Zhejiang Univ Sci B. 2012; 13(3):192-202.

27. Hsu C-K, Hsu MM-L, Lee JY-Y, Baima B, Sticherling M, Erbagci Z, et al. Demodicosis: A clinicopathological study. J Am Acad Dermatol. 2009; 60(3):453-62.

28. Moravvej H, Dehghan-Mangabadi M, Abbasian M-R, Meshkat-Razavi G. Association of rosacea with demodicosis. Arch Iran Med. 2007; 10(2):199-203.

29. Kim JHC, Chun YS, Kim JHC. Clinical and immunological responses in ocular demodecosis. J Korean Med Sci. 2011; 26(9):1231-7.

Revisión de pares: Recibido: 22 / 06 / 2019 Aceptado: 31/ 07/ 2019 